\title{
Endocrinopatie e anemie / anemie ed endocrinopatie: una si(pali)ndrome spesso misconosciuta
}

\author{
Tommaso Piticchio ${ }^{1}$. Francesco Frasca ${ }^{1}$
}

Accettato: 15 marzo 2020 / Pubblicato online: 12 ottobre 2020

(c) The Author(s) 2020

Sommario È comune osservazione nella pratica clinica che, spesso, i deficit endocrini si accompagnano ad anemie di vario grado. È anche noto che alcune specifiche anemie quali la talassemia possono essere associate a deficit endocrini di diversa gravità ed espressione. La gran parte degli ormoni, con vari meccanismi, può regolare la produzione di eritrociti nel midollo osseo. Quest'azione ormonale può essere diretta sui precursori della linea eritrocitaria o indiretta sulla produzione di eritropoietina a livello renale. Inoltre, quest'effetto può esplicarsi favorendo l'azione dell' eritropoietina sui precursori eritroidi stessi. In questa rassegna saranno presi in esame i diversi aspetti, onde includere l'anemia come parte integrante della pratica clinica endocrinologica sia dal punto di vista diagnostico che terapeutico.

Parole chiave Endocrinopatie - Anemia - Diabete mellito · Gastrite atrofica $\cdot$ Corticosteroidi $\cdot$ Eritropoietina

\section{Introduzione}

Da più di un secolo lo studio della fisiopatologia correlata alle varie endocrinopatie ha messo in luce un rapporto pressoché costante con le anemie. Rapporto che, a sua

Proposto da Rosaria M. Ruggeri.

Materiale elettronico supplementare La versione elettronica di questo articolo (https://doi.org/10.1007/s40619-020-00764-6) contiene materiale supplementare, disponibile per gli utenti autorizzati.

$凶$ F. Frasca

f.frasca@unict.it

1 Dipartimento di Medicina Clinica e Sperimentale, Università di Catania, Catania, Italia
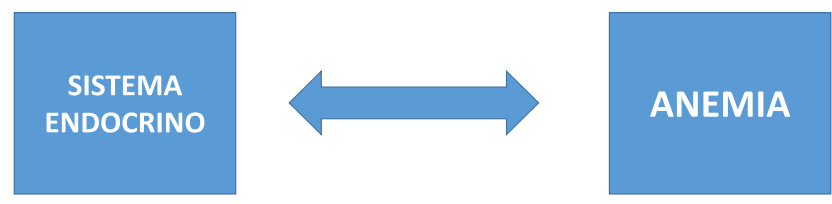

Fig. 1 La palindrome endocrinopatia - anemia - endocrinopatia

volta, può essere declinato in senso palindromo se partendo dall'anemia si va alla ricerca di un'eventuale endocrinopatia sottostante spesso misconosciuta, ad eccezione di casi paradigmatici come quelli dei pazienti talassemici (Fig. 1).

Gli ormoni intervengono sull'eritropoiesi stimolando la produzione di eritropoietina a livello renale, favorendo l'attività dell'eritropoietina stessa sui precursori eritroidi midollari e inducendo le cellule quiescenti del midollo osseo a entrare in fase cellulare (Fig. 2).

Inoltre, i processi autoimmunitari alla base di molteplici endocrinopatie e alcuni farmaci usati nella pratica clinica possono contestualmente interferire con l'assorbimento degli elementi fondamentali per la sintesi dei globuli rossi e con la loro emivita.

Infine, è bene sottolineare come, in virtù dei molteplici fattori in gioco, a una singola endocrinopatia possano associarsi più forme di anemia e come quest'ultima abbia un ruolo centrale nella progressione di complicanze e nella valutazione del compenso delle malattie endocrino-metaboliche.

\section{Endocrinopatie e anemie}

\section{Ipopituitarismo}

L'effetto del deficit di ormoni ipofisari sull'anemia si ricava in maniera analitica da alcuni modelli animali. L'ipofi- 
Fig. 2 Effetto degli ormoni sull'eritropoiesi. 1, diretto sui precursori eritroidi; 2 , indiretto sulla stimolazione della produzione renale di eritropoietina; 3 , indiretto sulla facilitazione dell'effetto dell'eritropoietina sui precursori eritroidi
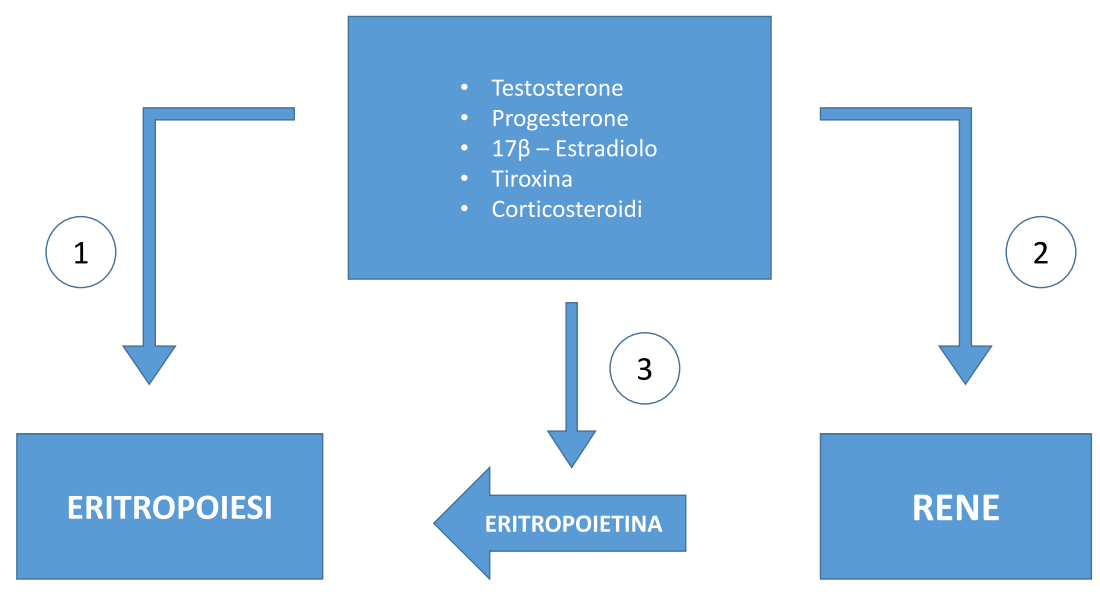

sectomia nel ratto è seguita da anemia da carenza eritroide moderata-grave, mentre la rimozione selettiva del lobo intermedio-posteriore non ha alcun effetto. In questo modello, poiché l'anemia da ipofisectomia è molto simile a quella della tiroidectomia, si ritiene che la carenza di TSH giochi un ruolo determinante. D'altra parte, negli animali ipofisectomizzati, l'eritropiesi è ripristinata completamente dalla contemporanea somministrazione di TSH e ACTH, oppure da tiroxina, glucocorticoidi e androgeni.

Sebbene il somatotropo, tramite l'IGF-I, abbia mostrato un effetto positivo sull'eritropoiesi in vitro, la carenza di GH di per sé non si associa ad anemia in vivo.

In linea con il modello animale, nei pazienti con ipopituitarismo o ipofisectomizzati, si osserva un'anemia normocitico/normocromica, con normale emivita delle emazie e ridotta produzione midollare. In questi soggetti l'anemia si corregge mediante la terapia sostitutiva tiroidea, surrenalica e gonadica.

\section{Disfunzioni tiroidee}

Nel 1881, Charcot osservava che i pazienti affetti da cretinismo e mixedema erano anche anemici. Al contempo, il chirurgo svizzero Kocher riportava che la tiroidectomia totale causava una riduzione della conta eritrocitaria.

Le caratteristiche dell'anemia associata all'ipotiroidismo sono state sempre oggetto di dibattito, poiché può avere presentazioni cliniche molto variabili (normocitica, micro o macrocitica). Parecchi studi hanno cercato di chiarire la patogenesi di questa anemia, cercando di distinguere l'effetto diretto degli ormoni tiroidei sull'eritropoiesi da altri fattori confondenti, quali carenza di ferro, vitamina B12 (VitB12) e acido folico che si associano spesso all'ipotiroidismo autoimmune. Gli studi finalizzati a studiare gli effetti diretti degli ormoni tiroidei sull'eritropoiesi sono stati condotti in animali tiroidectomizzati. In questi animali, il tasso di produzione eritrocitaria aumenta con la somministrazione di T4, T3 o estratti di tiroide secca e questo effetto sembra correlare con l'aumento del consumo di ossigeno indotto dagli ormoni tiroidei. Studi in vitro hanno inoltre dimostrato che gli ormoni tiroidei hanno un effetto diretto sull'eritropoiesi: da un lato l'ormone tiroideo aumenta la produzione di eritropoietina in risposta all'ipossia, dall'altro potenzia l'effetto dell'eritropoietina sulle colonie eritroidi $[1,2]$. Studi di cinetica del ferro, effettuati in questi animali, indicano che l'anemia è dunque su reticolocitopenia/ipoplasia della linea eritroide con emivita eritrocitaria normale.

L'anemia che si osserva nei pazienti ipotiroidei non è così definita, perché influenzata anche da possibili carenze nutrizionali associate. D'altra parte, molti soggetti ipotiroidei hanno un' anemia ipoplastica che è resistente alla terapia con ferro, vitamina B12 o acido folico, che è molto simile all' anemia che si osserva negli animali tiroidectomizzati. Come nel modello animale, nei pazienti ipotiroidei l'emivita degli eritrociti è normale come pure la velocità di utilizzo del ferro. Tuttavia, il livello di anemia dei soggetti ipotiroidei non è fedelmente rappresentato dall'ematocrito, in quanto in questi pazienti sussiste una riduzione del volume plasmatico. Infatti, immediatamente dopo l'inizio della terapia sostitutiva, in questi soggetti non è raro osservare un temporaneo peggioramento dell'ematocrito, perché il volume plasmatico si ripristina più velocemente della quota eritrocitaria. Sebbene la forma normocitica normocromica sia l'anemia tipica del deficit di ormoni tiroidei, la forma più frequente che si osserva in questi pazienti è quella microcitica ipocromica dovuta a carenza di ferro. Nelle donne, questa forma è dovuta all'ipocloridia e alle frequenti menometrorragie che spesso si associano all'ipotiroidismo [3], mentre negli uomini è dovuta soprattutto al ridotto assorbimento di ferro causato dall'ipocloridria. In questi pazienti, si può anche osservare, al contrario, un'anemia megaloblastica, legata alla carenza di vitamina B12 e/o acido folico. Com'è noto, gli ipotiroidei autoimmuni hanno un'elevata incidenza di anemia megaloblastica dovuta a carenza di fattore intrinseco per presenza di gastrite atrofica (10-40\% degli ipotiroidei autoimmuni) [4]. A questo si aggiunge spesso, se non costantemente, anche una significativa carenza di folati. 
Nonostante l'effetto positivo diretto degli ormoni tiroidei sulla eritropoiesi, molto raramente nella tireotossicosi si osserva poliglobulia. Questo fenomeno è dovuto in parte al fatto che l'aumento della gittata cardiaca viene incontro all'incremento nella richiesta tissutale di ossigeno senza un significativo aumento dell'attività eritropoietinica. Vari studi, tuttavia, dimostrano che nei soggetti ipertiroidei l'attività eritropoietica midollare è lievemente, ma significativamente, aumentata. Il fatto che in queste condizioni non si osservi una poliglobulia viene spiegato dal concomitante aumento del volume plasmatico. L'assenza di poliglobulia viene anche spiegata da studi sull'emivita eritrocitaria che ne dimostrano una durata lievemente ridotta in circolo a causa di concomitanti fenomeni autoimmuni. Al contrario, in alcuni casi di ipertiroidismo si è potuta osservare una concomitante anemia, sia per la presenza di autoimmunità gastrica che di anticorpi anti-eritropoietina.

\section{Disfunzioni surrenaliche}

Negli animali da esperimento, l'adrenalectomia causa una moderata anemia, responsiva alla terapia con glucocorticoidi o eritropoietina. Nei pazienti con Morbo di Addison si osserva un'anemia normocitico/normocromica simile a quella del modello animale. L'anemia dell'iposurrenalico viene spesso sottovalutata a causa della concomitante riduzione del volume plasmatico, la quale altera la concentrazione emoglobinica e l'ematocrito che, dunque, non riflettono la riduzione del volume eritrocitario. Il ruolo dei glucocorticoidi in quest'ambito, tuttavia, non è facilmente valutabile, in quanto concentrazioni fisiologiche di questi ormoni causano solo lievi effetti sull'eritropoiesi. A dosi farmacologiche i glucocorticoidi causano una modesta eritrocitosi a un livello simile a quella osservata nella Sindrome di Cushing e, occasionalmente, nella Sindrome di Bartter (iperaldosternismo compensatorio). In ogni caso, non è stato ancora ulteriormente chiarito se l'effetto degli steroidi surrenalici è diretto sui precursori eritroidi o mediata dall'eritropoietina.

\section{Disfunzioni gonadiche e anemia}

L'effetto positivo degli androgeni sull'eritropoiesi è stato ben documentato in vari sistemi biologici, sia a livelli fisiologici che farmacologici, e utilizzato addirittura nella terapia di varie forme di anemia indipendentemente dalla causa. Nell' animale maschio da esperimento la castrazione causa una riduzione della produzione di eritrociti, del livello di emoglobina e concentrazione eritrocitaria simile a quella della femmina fertile. Nei soggetti umani maschi adulti, sessualmente maturi, i livelli di emoglobina sono in genere 1-2 g/dl più elevati rispetto l'infanzia, l'anzianità, l'orchiectomia, o l'ipogonadismo. In queste condizioni, i livelli di emoglobina sono simili ai soggetti di sesso femminile di pari età. Gli androgeni a dosi farmacologiche stimolano la produzione di globuli rossi mediante un significativo aumento dei livelli di eritropoietina, nonché potenziando l'effetto della stessa sul midollo osseo. Studi in vitro hanno dimostrato che gli isomeri androgenici hanno due differenti attività biologiche: l'isomero $5 \alpha-\mathrm{H}$ è androgenizzante e causa il rilascio dell'eritropoietina nel rene, l'isomero $5 \beta$-H non è androgenizzante ma stimola le cellule quiescenti del midollo osseo a entrare nella fase cellulare, sensibile allo stimolo eritropoietinico. Infatti, prima dell'introduzione dell'eritropoietina ricombinante l'anemia dell'insufficienza renale veniva curata con gli androgeni in entrambi i sessi e tutt'oggi in molti paesi gli androgeni vengono utilizzati in combinazione con l'eritropoietina per ridurne le quantità e di conseguenza i costi.

Studi sugli effetti fisiologici degli estrogeni indicano un lieve effetto soppressivo sull'eritropoiesi e, a dosi farmacologiche, possono causare una modesta anemia, ma non è ancora chiaro se ciò possa avvenire attraverso la soppressione della produzione di eritropoietina o direttamente inibendo l'effetto della eritropoietina sui precursori eritroidi.

\section{Disfunzioni paratiroidee e anemia}

Nell'iperparatiroidismo primitivo si può talvolta osservare anemia che si risolve con la rimozione dell'adenoma paratiroideo [5]. Inoltre, la paratiroidectomia nell'insufficienza renale cronica causa un miglioramento dell'anemia. Tuttavia, un effetto soppressivo diretto del paratormone sulla linea eritroide non è stato mai definitivamente dimostrato. Queste osservazioni indicano che l'anemia associata all'iperparatiroidismo primitivo/secondario è indirettamente causata sia dal danno renale (nefroalcinosi) che dalla sclerosi del midollo osseo, con riduzione della proliferazione dei precursori eritroidi.

\section{Diabete mellito e anemia}

La coesistenza di anemia nel diabete mellito è un'evidenza clinica in aumento. Si calcola che la prevalenza di anemia nei soggetti diabetici sia del $14-45 \%$ tra le varie etnie $[6,7]$. Infatti, nei pazienti diabetici il rischio di anemia è circa 2-3 volte più alto che nei soggetti non diabetici. Ovviamente, il rischio di sviluppare anemia nei diabetici è associato con la progressione del danno renale. Tuttavia, i soggetti con nefropatia diabetica hanno un rischio più alto di anemia rispetto i nefropatici da altra causa. Inoltre, il fatto che l'anemia può insorgere nei diabetici prima dell'evidenza del danno renale suggerisce che possano esistere altre cause. Esiste anche una correlazione tra cattivo compenso glico-metabolico e anemia e tale rischio aumenta con l'insorgenza della malattia renale. D'altra parte, la presenza di anemia nel diabetico aumenta il rischio di progressione delle complicanze micro e macrovascolari. Pertanto, l'anemia nei diabetici 
Fig. 3 Meccanismi patogenetici dell'anemia nel diabetico

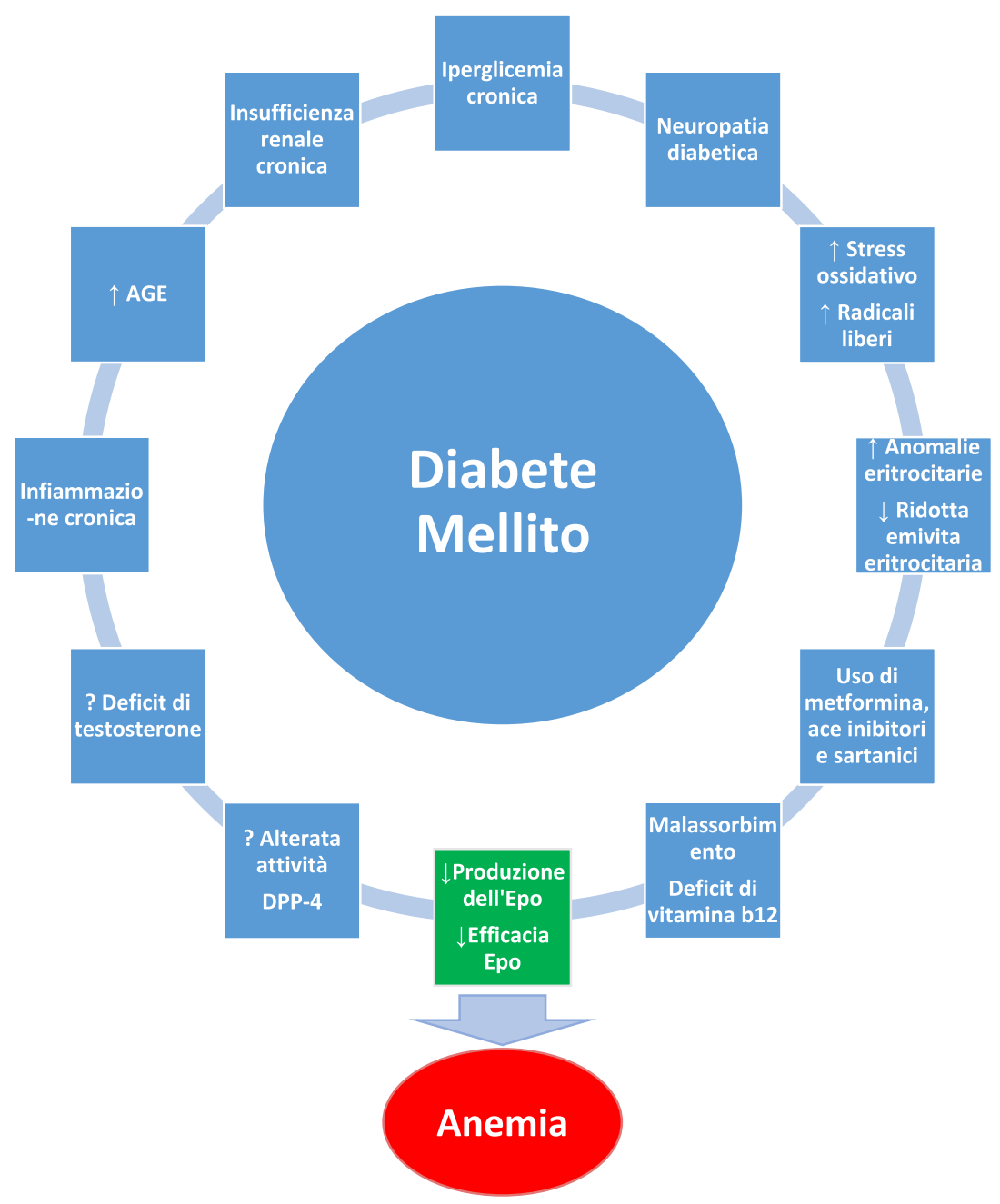

va diagnosticata e trattata alla stregua degli altri fattori di rischio.

\section{Anemia nel diabete tipo 1 (DT1)}

L'anemia nel diabete tipo 1 è causata dalle patologie autoimmuni concomitanti tra cui la gastrite autoimmune. In questi pazienti spesso si osserva un'anemia sideropenica che precede quella megaloblastica. In alcuni casi, le due anemie coesistono rendendo il quadro ancora più complesso. La patogenesi è legata al fatto che la ipo/acloridria della gastrite autoimmune porta a una riduzione nelle fasi precoci dell'attività $\mathrm{H}+/ \mathrm{K}+\mathrm{ATP}$-asica con conseguente ridotto assorbimento di ferro. Nelle fasi più avanzate, la distruzione delle cellule parietali gastriche causa la riduzione di produzione di fattore intrinseco con conseguente ridotto assorbimento di VitB12 e anemia megaloblastica. Inoltre, il diabete tipo 1 è spesso associato a disturbi che provocano malassorbimento quali la celiachia, che causa un'ipotrofia dei villi con ulteriore riduzione di assorbimento di ferro e VitB12. Un'altra causa di anemia nei pazienti con DT1 è l'ipotiroidismo: il 10-30\% dei pazienti con DT1 hanno tiroidite di Hashimoto con conseguente ipotiroidismo, che causa un'anemia normocitica normocromica come descritto in precedenza.

\section{Anemia nel diabete Tipo 2 (DT2)}

La patogenesi dell'anemia nel DT2 è multifattoriale e complessa (Fig. 3). A parte l'insufficienza renale, in questa patologia possono coesistere la carenza di ferro e una iporesponsività all'eritropietina dovuta alla sua glicazione e del suo recettore. Inoltre, in questi pazienti la neuropatia autonomica porta a una riduzione della stimolazione adrenergica per la produzione di eritropoietina. Altro fattore che concorre all'anemia in questi pazienti è l'ipogonadismo con ridotti livelli di testosterone. Inoltre, la produzione di AGE nei diabetici scompensati sembra essere coinvolta nella ridotta emivita degli eritrociti. D'altra parte, la presenza di elevati livelli di citochine pro-infiammatorie (IL-1, 6, TNF- $\alpha, \beta$ e IFN) sembra favorire l'apoptosi dei precursori eritroidi. Altro fattore aggravante è l'aumentata proteinuria ed eliminazione dell'eritropoietina con le urine. 
Tabella 1 Effetto degli ipoglicemizzanti orali sull'anemia del diabetico

metformina

tiazolidinedioni

inibitori del DPP4

glifozine

\section{Farmaci del DT2}

La terapia con ipoglicemizzanti orali del DT2 può giocare anche un ruolo importante nel contribuire all'insorgenza di anemia (Tabella 1). Tra questi, la più usata è la metformina che notoriamente causa un ridotto assorbimento di VitB12 dopo in media quattro mesi di somministrazione. Tuttavia, l'anemia megaloblastica può esordire dopo 5-10 anni di terapia con metformina a causa delle riserve epatiche di VitB12. In generale, si può affermare che circa il 10$30 \%$ dei pazienti che assumono metformina da lungo tempo mostra malassorbimento di VitB12 e il rischio di carenza di VitB12 raddoppia a tre anni dall'inizio della terapia. Anche i tiazolidinedioni possono contribuire all'anemia del diabetico con diversi meccanismi che comprendono l'espansione del liquido extracellulare e l'accumulo di grasso nel midollo osseo con riduzione della massa eritroide. Al contrario, gli inibitori del DPP4 migliorano l'anemia aumentando i livelli di eritropoietina a causa della riduzione della sua degradazione da parte del DPP-4. Infatti, l'uso degli inibitori del DPP-4 riduce il fabbisogno degli erythropoietin stimulating agents (ESA) nei pazienti diabetici dializzati. Anche gli inibitori dell'SGLT2 migliorano l'anemia nel DT2, aumentando i livelli di eritropoietina attraverso il loro effetto nefroprotettivo, inibendo lo stress ossidativo e l'ipossia tubulo-interstiziale.

\section{Anemia e complicanze del diabete}

L'anemia del diabetico, causata in parte dall'insufficienza renale, può a sua volta influenzare la progressione della nefropatia diabetica, favorendo un circolo vizioso verso l'insufficienza renale terminale. L'anemia, infatti, aumenta la pressione glomerulare e la proteinuria per aumento dell' attività adrenergica.

L'anemia aumenta il rischio di retinopatia diabetica (rischio raddoppiato per un'emoglobina $<12 \mathrm{~g} / \mathrm{dl}$ ) tramite l'aggravamento dell'ipossia retinica con conseguente incremento di produzione di VEGF e, di conseguenza, formazione di neovasi [8].

L'anemia nel diabetico è anche un fattore di rischio indipendente per mortalità cardiovascolare e si associa a ipertrofia del ventricolo sinistro e scompenso cardiaco. Questi danni cardiaci sono dovuti all'effetto dell'anemia sulle modificazioni emodinamiche, nonché sulla carenza di eritropo-
Tabella 2 Malattie endocrine e anemia

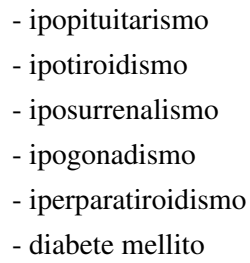

ietina sul rimodellamento del muscolo cardiaco. Anche il rischio di ulcere diabetiche e amputazioni è aumentato dall'anemia, a causa della ridotta ossigenazione cutanea e ridotta guarigione delle ferite.

La correzione dell' anemia nei pazienti diabetici, indipendentemente dalla presenza di danno renale, è stata dimostrata efficace nel ridurre l'insorgenza di complicanze e di mortalità. La terapia va effettuata sulla base del meccanismo patogenetico prevalente dell'anemia. Nei pazienti con danno renale è stato dimostrato che un' emoglobina di 11-12 g/dl è sufficiente per migliorare l'evoluzione delle complicanze. Al contrario, un' emoglobina maggiore di $13 \mathrm{~g} / \mathrm{dl}$ aumenta il rischio cardiovascolare. La correzione dell'anemia è anche importante ai fini del monitoraggio del compenso glicemico. Va infatti considerato che l'anemia da carenza di ferro o VitB12, causando un ridotto turnover degli eritrociti, provoca un'esposizione maggiore dell'emoglobina alla glicazione e, quindi, a valori di glicata falsamente elevati (Tabella 2).

\section{Anemie ed endocrinopatie}

\section{Talassemia}

Negli ultimi decenni, i soggetti talassemici mostrano una sopravvivenza più lunga a causa del miglioramento delle cure. Pertanto, soffrono frequentemente di ipofunzioni endocrine a causa del danno dovuto all' accumulo di ferro nei parenchimi ghiandolari (Fig. 4). La disfunzione più frequente (40$50 \%$ dei casi) è l'ipogonadismo ipogonadotropo per deficit di LH e FSH (Fig. 5). Solo occasionalmente l'ipogonadismo può essere primitivo $\mathrm{e}$, in genere, si manifesta nelle donne verso i 30 anni di età, quando la vascolarizzazione ovarica è massimale [9]. L'esordio in genere è un quadro di pubertà ritardata o assente. Il deficit di $\mathrm{GH}$ è secondo in ordine di frequenza (15-20\% dei casi) e si manifesta con ritardo di crescita. Altro deficit abbastanza frequente è a carico del metabolismo glicidico: IGT e diabete mellito si osserva in circa il $10 \%$ dei soggetti. In questi soggetti, l'accumulo di ferro causa diabete con meccanismo combinato: insulinoresistenza epatica per accumulo di ferro e successivamente deficit secretorio delle $\beta$-cellule per lo stesso motivo. Infatti, questi soggetti sono negativi per gli anticorpi anti $\beta$-cellule, non sono proni alla chetosi e hanno un'elevata soglia renale 
Fig. 4 Disordini endocrini nella talassemia: il sovraccarico di ferro nei parenchimi ghiandolari causa deficit. Il contemporaneo accumulo di ferro nel fegato e nel pancreas contribuisce all'insorgenza del diabete. $N T B I$, non-transferrin-bound serum iron

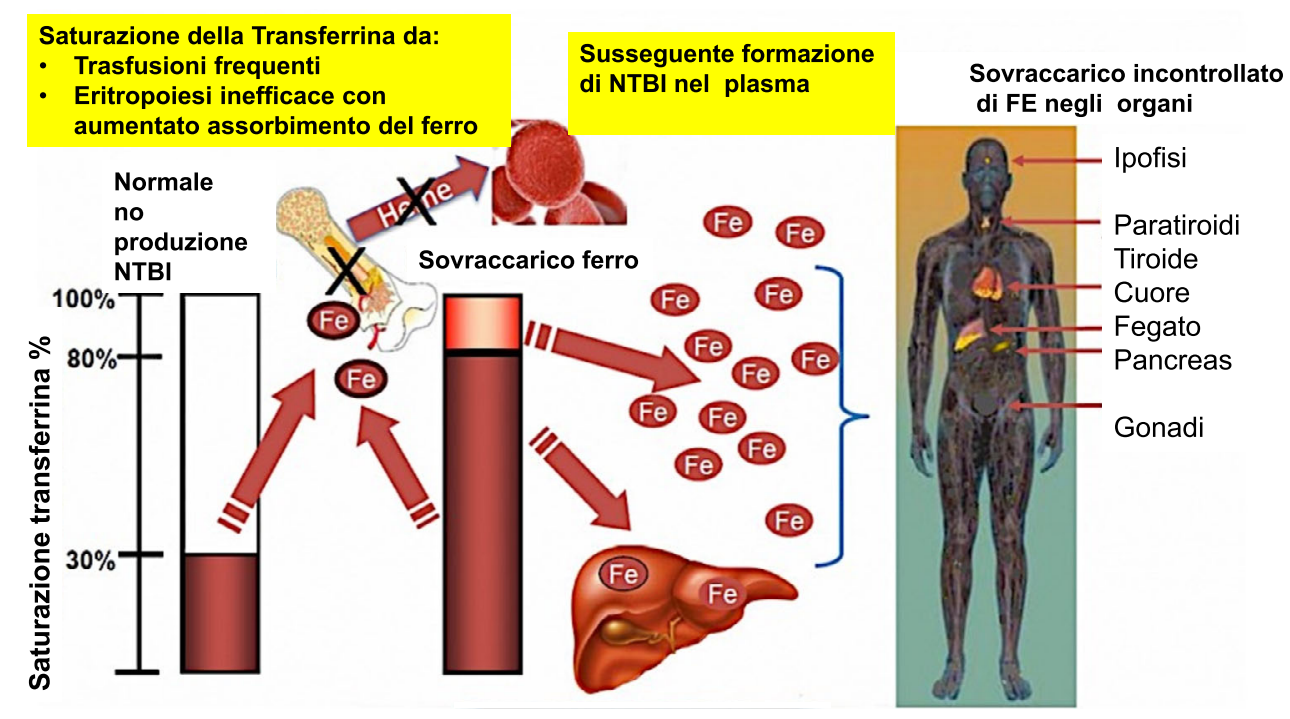

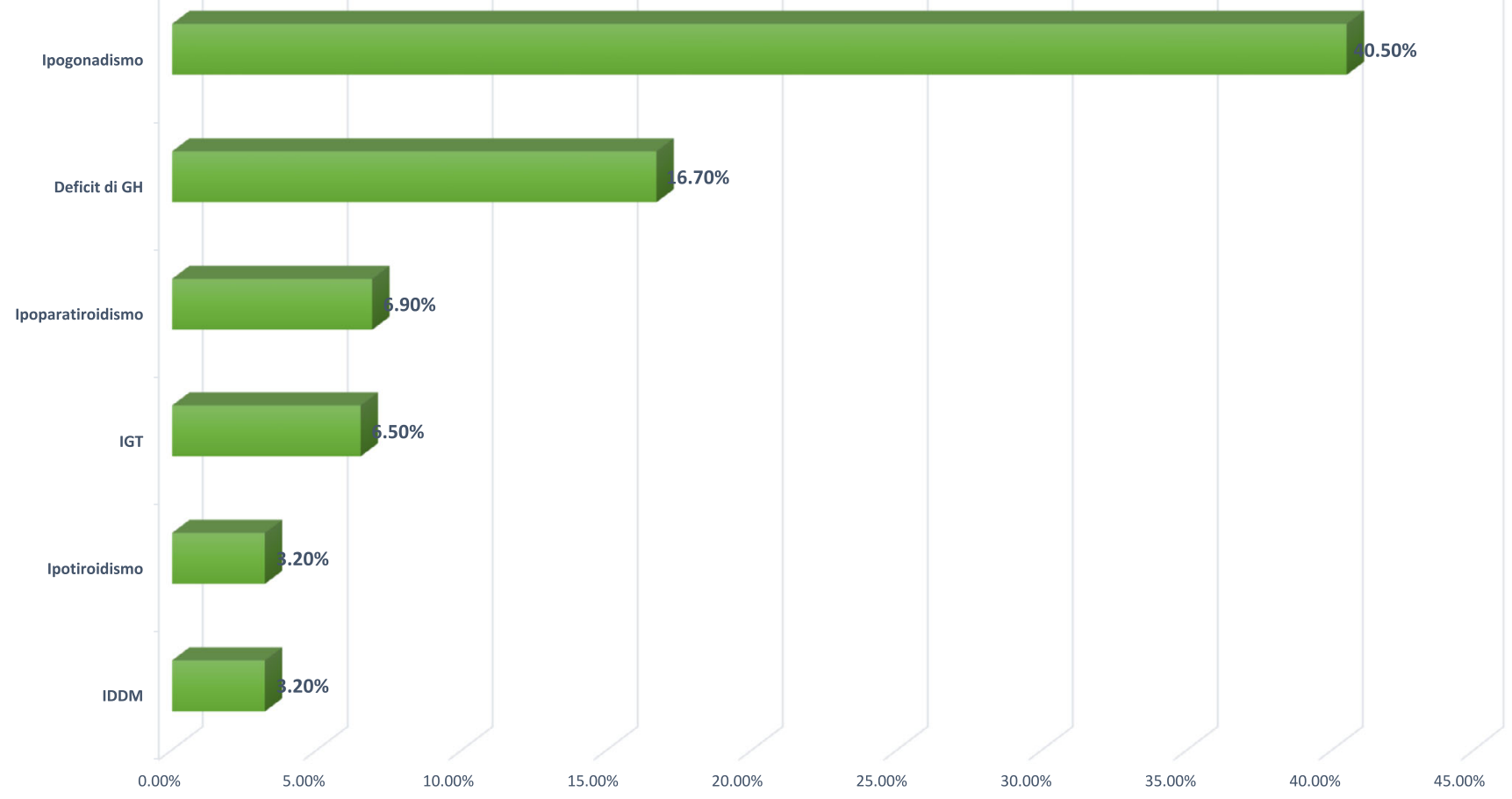

Fig. 5 Frequenza dei deficit endocrini nel talassemico

al glucosio $[10,11]$. Altra alterazione endocrina abbastanza frequente è l'ipoparatiroidismo (5-10\% dei casi) e ha un decorso spesso asintomatico. La diagnosi viene posta in seguito a riscontro occasionale di ipocalcemia. Un'alterazione endocrina poco frequente è l'ipotiroidismo primitivo (2-5\% dei casi). Viene scoperto con il dosaggio di FT4 e TSH. Le forme di ipotirodismo subclinico possono essere reversibili ottimizzando la terapia chelante il ferro. Il deficit di LH e GH può essere accompagnato da deficit di altre tropine ipofisarie, per cui si può più raramente configurare un quadro di ipotiroidismo e iposurrenalismo centrali. L'iposurrenalismo con carenza di androgeni surrenalici può concorrere insieme all'ipogonadismo allo scarso sviluppo dei caratteri sessuali secondari (peli pubici e ascellari). Il quadro dell'iposurrenalismo è spesso asintomatico, la diagnosi va posta con test all'ACTH in pazienti astenici con decremento ponderale, sintomi peraltro comuni nella talassemia. La combinazione di deficit primitivi e secondari in questi pazienti può rendere difficile la diagnosi in alcune forme e la instaurazione di adeguata terapia sostitutiva [12]. 


\section{Anemia di Fanconi}

Sindrome genetica associata ad alterazioni dei meccanismi del riparo al danno al DNA, con instabilità cromosomica, aplasia midollare, difetti dello sviluppo e predisposizione a sviluppare tumori. Si associa a diversi difetti endocrini tra cui deficit di GH (50\% dei casi) ipotiroidismo (30-40\% dei casi), IGT (40\%), ipogonadismo (60\%), osteoporosi (80\%). Sebbene la prognosi non sia favorevole sul lungo termine, la diagnosi dei deficit e la conseguente terapia sostitutiva è necessaria per la qualità della vita $[13,14]$.

\section{Conclusioni}

L'anemia è un elemento che va sempre considerato nell'ambito della valutazione del paziente endocrinopatico, in quanto possibile conseguenza dell'endocrinopatia stessa. Essa va rivalutata dopo la correzione del deficit ormonale e inclusa come elemento responsabile della sintomatologia del paziente e della sua remissione. Va evitato il suo trattamento alla diagnosi del deficit endocrino; piuttosto, va trattata al ristabilirsi dei normali livelli ormonali, se persiste. Nel paziente endocrinopatico vanno valutate, inoltre, le concause dell'anemia ed eventualmente corrette (deficit di VitB12, folati, ferro). Nel paziente diabetico l'anemia deve far parte della valutazione delle complicanze in quanto la sua presenza è indice indiretto sia di cattivo compenso, che rischio ulteriore di progressione delle complicanze. Pertanto va corretta in base al meccanismo patogenetico prevalente. Attenzione particolare va posta all'effetto positivo e negativo della terapia con ipoglicemizzanti orali sull' anemia. Infine, occorre tener presente che un'anemia da cause inspiegabili e resistente alle comuni terapie potrebbe sottendere un'endocrinopatia misconosciuta. Pochi studi e linee guida sono presenti in questo ambito; pertanto, ulteriori ricerche nel settore andrebbero incoraggiate.

Funding Note Open access funding provided by Università degli Studi di Catania within the CRUI-CARE Agreement.

Conflitto di interesse Gli autori Tommaso Piticchio e Francesco Frasca dichiarano di non avere conflitti di interesse.

Consenso informato Lo studio presentato in questo articolo non ha richiesto sperimentazione umana.

Studi sugli animali Gli autori di questo articolo non hanno eseguito studi sugli animali.
Nota della casa editrice Springer Nature rimane neutrale in riguardo alle rivendicazioni giurisdizionali nelle mappe pubblicate e nelle affiliazioni istituzionali.

Open Access This article is licensed under a Creative Commons Attribution 4.0 International License, which permits use, sharing, adaptation, distribution and reproduction in any medium or format, as long as you give appropriate credit to the original author(s) and the source, provide a link to the Creative Commons licence, and indicate if changes were made. The images or other third party material in this article are included in the article's Creative Commons licence, unless indicated otherwise in a credit line to the material. If material is not included in the article's Creative Commons licence and your intended use is not permitted by statutory regulation or exceeds the permitted use, you will need to obtain permission directly from the copyright holder. To view a copy of this licence, visit http://creativecommons.org/licenses/by/4.0/.

\section{Bibliografia}

1. Golde DW, Bersch N, Chopra IJ, Cline MJ (1977) Thyroid hormones stimulate erythropoiesis in vitro. $\mathrm{Br} \mathrm{J}$ Haematol 37(2):173-177

2. Fandrey J, Pagel H, Frede S et al (1994) Thyroid hormones enhance hypoxia-induced erythropoietin production in vitro. Exp Hematol 22(3):272-277

3. Potaczek DP, Jankowska EA, Wypasek E, Undas A (2016) Iron deficiency: a novel risk factor of recurrence in patients after unprovoked venous thromboembolism. Pol Arch Med Wewn 126(3):159-165

4. Szczepanek-Parulska E, Hernik A, Ruchała M (2017) Anemia in thyroid diseases. Pol Arch Intern Med 127(5):352-360

5. Boxer M, Ellman L, Geller R, Wang CA (1977) Anemia in primary hyperparathyroidism. Arch Intern Med 137(5):588-593

6. Loutradis C, Skodra A, Georgianos P et al (2016) Diabetes mellitus increases the prevalence of anemia in patients with chronic kidney disease: a nested case-control study. World J Nephrol 5(4):358-366

7. Astor BC, Muntner P, Levin A et al (2002) Association of kidney function with anemia: the Third National Health and Nutrition Examination Survey (1988-1994). Arch Intern Med 162(12):1401-1408

8. Sahay M, Kalra S, Badani R et al (2017) Diabetes and anemia: International Diabetes Federation (IDF) - Southeast Asian Region (SEAR) position statement. Diabetes Metab Syndr 11(Suppl 2):S685-S695

9. Toumba M, Kanaris C, Simamonian K, Skordis N (2008) Outcome and management of pregnancy in women with thalassaemia in Cyprus. East Mediterr Health J 14(3):628-635

10. Wonke B, De Sanctis V (2001) Clinical aspects of transfusional iron overload. Clin Exp Hematol 12:322-334

11. De Sanctis V, Wonke B (1998) Growth and endocrine complications in thalassaemia Mediprint, Rome

12. El Kholy M, Elsedfy H, Soliman A (2014) Towards an optimization of the management of endocrine complications of thalassemia. J Pediatr Endocrinol Metab 27(9-10):801-805

13. Petryk A, Kanakatti Shankar R, Giri N et al (2015) Endocrine disorders in Fanconi anemia: recommendations for screening and treatment. J Clin Endocrinol Metab 100(3):803-811

14. Ruggeri RM, Giuffrida G, Campennì A (2018) Autoimmune endocrine diseases. Minerva Endocrinol 43(3):305-322 